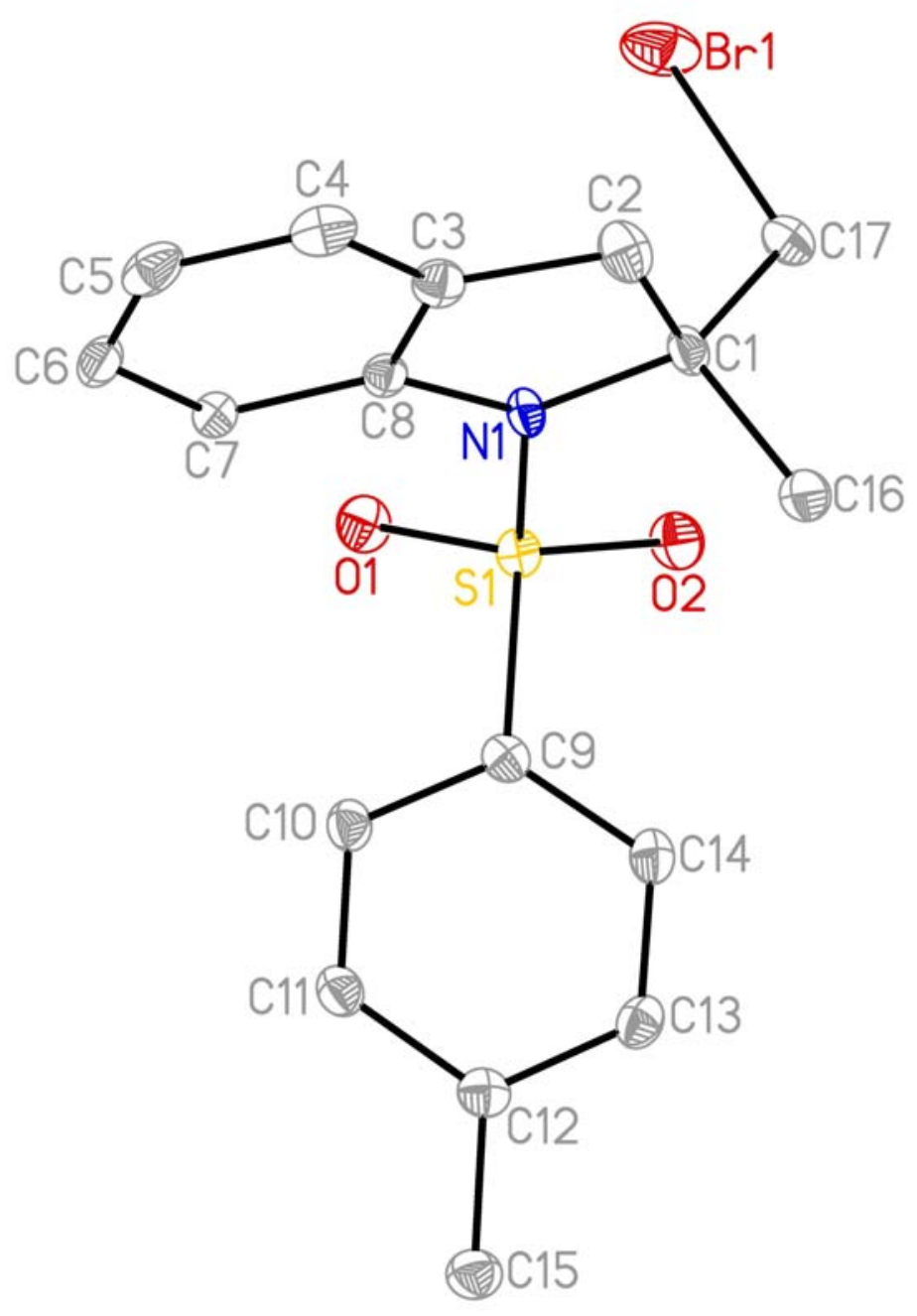

Figure 1. Molecular structure of the compound (ellipsoids are drawn with 50\% probability).

The structure determination and the report are by Oksana O. Gerlits.

\title{
Experimental Part.
}

X-ray diffraction data on 1 were collected at 90(1)K using a Bruker SMART1000 CCD diffractometer installed at a rotating anode source (MoK $\alpha$ radiation, $\lambda=0.71073 \AA$ ), and equipped with an Oxford Cryosystems nitrogen gas-flow apparatus. The data were collected by the rotation method with $0.3^{\circ}$ frame-width ( $\omega$ scan) and 20 sec exposure time per frame. Four sets of data (600 frames in each set) were collected, nominally covering half of reciprocal space. The data were integrated, scaled, sorted and averaged 
using the SMART software package. ${ }^{1}$ The structure was solved by Direct methods using SHELXTL NT Version $5.10 .^{2}$ The structure was refined by full-matrix least squares against $\mathrm{F}^{2}$.

Non-hydrogen atoms were refined anisotropically. Positions of hydrogen atoms were found by difference electron density Fourier synthesis. The $\mathrm{CH}_{3}$ hydrogens were treated as part of idealized $\mathrm{CH}_{3}$ groups with $\mathrm{U}_{\text {iso }}=1.5 \mathrm{U}_{\mathrm{eq}}$, while the remainder of the hydrogen atoms were refined with the "riding" model with $\mathrm{U}_{\mathrm{iso}}=1.2 \mathrm{U}_{\mathrm{eq}}$.

Atomic coordinates, anisotropic displacement parameters, bond lengths and angles are given in Tables 2, 3, 4 respectively. 
Table 1. Crystallographic data.

\begin{tabular}{|c|c|}
\hline Compound & 1 \\
\hline Formula & C17 H18 Br N O2 S \\
\hline $\mathrm{M}_{\mathrm{r}}\left[\mathrm{g} \cdot \mathrm{mol}^{-1}\right]$ & 380.29 \\
\hline Crystal shape/color & parallelepipeds/colorless \\
\hline Crystal system & Monoclinic \\
\hline Space group & $\mathrm{P} 2{ }_{1} / \mathrm{n}$ \\
\hline$a[\AA]$ & $11.5532(4)$ \\
\hline$b[\AA]$ & $11.4494(4)$ \\
\hline$c[\AA]$ & $12.2883(4)$ \\
\hline$\alpha\left[^{\circ}\right]$ & 90 \\
\hline$\beta\left[^{\circ}\right]$ & $96.685(1)$ \\
\hline$\gamma\left[^{\circ}\right]$ & 90 \\
\hline $\mathrm{V}\left[\AA^{3}\right]$ & $1614.4(1)$ \\
\hline $\mathrm{Z}$ & 4 \\
\hline$\rho_{\text {calc }}\left[\mathrm{g} / \mathrm{cm}^{3}\right]$ & 1.565 \\
\hline$\mu\left[\mathrm{mm}^{-1}\right]$ & 2.682 \\
\hline $\mathrm{T}[\mathrm{K}]$ & $90(1)$ \\
\hline Max. $2 \theta\left[^{\circ}\right]$ & 56.62 \\
\hline Absorption correction method & SADABS 2.05 \\
\hline Reflections measured & 19441 \\
\hline Unique reflections $\left(\mathrm{R}_{\text {int }}\right)$ & $4016(0.0332)$ \\
\hline Reflections $\mathrm{I}>4 \sigma(\mathrm{I})$ & 3458 \\
\hline Parameters refined & 201 \\
\hline $\mathrm{R}[\mathrm{I}>2 \sigma(\mathrm{I})]$ & 0.0310 \\
\hline $\mathrm{wR}_{2}$ & 0.0816 \\
\hline G.O.F. & 1.075 \\
\hline
\end{tabular}


Table 2. Atomic coordinates $\left(10^{4}\right)$ and equivalent isotropic displacement parameters $\left(\AA^{2} \times 10^{3}\right)$.

\begin{tabular}{|c|c|c|c|}
\hline Atom & $\mathrm{x}$ & $\mathrm{y}$ & $\mathrm{U}(\mathrm{eq})$ \\
\hline $\begin{array}{l}\operatorname{Br}(1) \\
29(1)\end{array}$ & $1704(1)$ & $4058(1)$ & $995(1)$ \\
\hline $\begin{array}{l}S(1) \\
13(1)\end{array}$ & $5172(1)$ & $3182(1)$ & $1806(1)$ \\
\hline $\begin{array}{l}O(1) \\
18(1)\end{array}$ & $4974(1)$ & $1951(1)$ & $1908(1)$ \\
\hline $\begin{array}{l}O(2) \\
19(1)\end{array}$ & $5090(1)$ & $3702(1)$ & $738(1)$ \\
\hline $\begin{array}{l}N(1) \\
13(1)\end{array}$ & $4206(2)$ & $3845(1)$ & $2463(1)$ \\
\hline $\begin{array}{l}C(1) \\
15(1)\end{array}$ & $3779(2)$ & $5079(2)$ & $2216(2)$ \\
\hline $\begin{array}{l}C(2) \\
20(1)\end{array}$ & $3099(2)$ & $5371(2)$ & $3200(2)$ \\
\hline $\begin{array}{l}C(3) \\
16(1)\end{array}$ & $3318(2)$ & $4367(2)$ & $3977(2)$ \\
\hline $\begin{array}{l}C(4) \\
20(1)\end{array}$ & $2955(2)$ & $4217(2)$ & $5002(2)$ \\
\hline $\begin{array}{l}C(5) \\
22(1)\end{array}$ & $3221(2)$ & $3181(2)$ & $5572(2)$ \\
\hline $\begin{array}{l}C(6) \\
19(1)\end{array}$ & $3855(2)$ & $2316(2)$ & $5113(2)$ \\
\hline $\begin{array}{l}C(7) \\
15(1)\end{array}$ & $4222(2)$ & $2453(2)$ & $4080(2)$ \\
\hline $\begin{array}{l}C(8) \\
13(1)\end{array}$ & $3951(2)$ & 3496 (2) & $3524(2)$ \\
\hline $\begin{array}{l}C(9) \\
14(1)\end{array}$ & $6584(2)$ & $3472(2)$ & $2468(2)$ \\
\hline $\begin{array}{l}C(10) \\
15(1)\end{array}$ & $7047(2)$ & $2789(2)$ & $3352(2)$ \\
\hline $\begin{array}{l}C(11) \\
16(1)\end{array}$ & $8187(2)$ & $2974(2)$ & $3814(2)$ \\
\hline $\begin{array}{l}C(12) \\
15(1)\end{array}$ & $8888(2)$ & $3823(2)$ & $3404(2)$ \\
\hline $\begin{array}{l}C(13) \\
16(1)\end{array}$ & $8400(2)$ & $4510(2)$ & $2526(2)$ \\
\hline $\begin{array}{l}C(14) \\
16(1)\end{array}$ & $7263(2)$ & $4340(2)$ & $2055(2)$ \\
\hline $\begin{array}{l}C(15) \\
19(1)\end{array}$ & $10137(2)$ & $4004(2)$ & $3877(2)$ \\
\hline $\begin{array}{l}C(16) \\
26(1)\end{array}$ & $4777(2)$ & $5954(2)$ & $2166(2)$ \\
\hline $\begin{array}{l}C(17) \\
21(1)\end{array}$ & $3021(2)$ & $5137(2)$ & $1121(2)$ \\
\hline
\end{tabular}


Table 3. Anisotropic displacement parameters $\left(\AA^{2} \times 10^{3}\right)$.

\begin{tabular}{|c|c|c|c|c|c|}
\hline $\begin{array}{l}\text { Atom } \\
\text { U12 }\end{array}$ & U11 & $\mathrm{U} 22$ & U33 & U23 & U13 \\
\hline $\begin{array}{l}\operatorname{Br}(1) \\
-2(1)\end{array}$ & $18(1)$ & $30(1)$ & $37(1)$ & $-9(1)$ & $-7(1)$ \\
\hline $\begin{array}{l}S(1) \\
0(1)\end{array}$ & $15(1)$ & $12(1)$ & 11 (1) & $-1(1)$ & $1(1)$ \\
\hline $\begin{array}{l}O(1) \\
-1(1)\end{array}$ & $21(1)$ & $12(1)$ & 21 (1) & $-4(1)$ & $2(1)$ \\
\hline $\begin{array}{l}O(2) \\
O(1)\end{array}$ & 21 (1) & $23(1)$ & 11 (1) & $0(1)$ & $0(1)$ \\
\hline $\begin{array}{l}\mathrm{N}(1) \\
2(1)\end{array}$ & $15(1)$ & $10(1)$ & $13(1)$ & $2(1)$ & $2(1)$ \\
\hline $\begin{array}{l}C(1) \\
1(1)\end{array}$ & $16(1)$ & $10(1)$ & $18(1)$ & $2(1)$ & $-1(1)$ \\
\hline $\begin{array}{l}C(2) \\
6(1)\end{array}$ & $25(1)$ & $15(1)$ & $20(1)$ & $-2(1)$ & $3(1)$ \\
\hline $\begin{array}{l}C(3) \\
-2(1)\end{array}$ & $15(1)$ & $16(1)$ & $17(1)$ & $-2(1)$ & $0(1)$ \\
\hline $\begin{array}{l}C(4) \\
-2(1)\end{array}$ & $17(1)$ & $25(1)$ & $19(1)$ & $-8(1)$ & $4(1)$ \\
\hline $\begin{array}{l}C(5) \\
-10(1)\end{array}$ & $20(1)$ & $33(1)$ & $13(1)$ & $0(1)$ & $2(1)$ \\
\hline $\begin{array}{l}C(6) \\
-7(1)\end{array}$ & $17(1)$ & $23(1)$ & $17(1)$ & $7(1)$ & $-1(1)$ \\
\hline $\begin{array}{l}C(7) \\
-1(1)\end{array}$ & $14(1)$ & $16(1)$ & $17(1)$ & $3(1)$ & $1(1)$ \\
\hline $\begin{array}{l}C(8) \\
-2(1)\end{array}$ & $12(1)$ & $14(1)$ & $12(1)$ & $0(1)$ & $0(1)$ \\
\hline $\begin{array}{l}C(9) \\
2(1)\end{array}$ & $15(1)$ & $14(1)$ & $13(1)$ & $-2(1)$ & $2(1)$ \\
\hline $\begin{array}{l}C(10) \\
2(1)\end{array}$ & $17(1)$ & $13(1)$ & $17(1)$ & $1(1)$ & $4(1)$ \\
\hline $\begin{array}{l}C(11) \\
3(1)\end{array}$ & $17(1)$ & $15(1)$ & $17(1)$ & $1(1)$ & $2(1)$ \\
\hline $\begin{array}{l}C(12) \\
2(1)\end{array}$ & $16(1)$ & $13(1)$ & $17(1)$ & $-4(1)$ & $3(1)$ \\
\hline $\begin{array}{l}C(13) \\
-1(1)\end{array}$ & $19(1)$ & $13(1)$ & $18(1)$ & $0(1)$ & $6(1)$ \\
\hline $\begin{array}{l}C(14) \\
1(1)\end{array}$ & $20(1)$ & $15(1)$ & $15(1)$ & $2(1)$ & $3(1)$ \\
\hline $\begin{array}{l}C(15) \\
-1(1)\end{array}$ & $16(1)$ & $17(1)$ & $25(1)$ & $-1(1)$ & $1(1)$ \\
\hline $\begin{array}{l}C(16) \\
1(1)\end{array}$ & $17(1)$ & $17(1)$ & $43(1)$ & $9(1)$ & $2(1)$ \\
\hline $\begin{array}{l}C(17) \\
1(1)\end{array}$ & $16(1)$ & $25(1)$ & $20(1)$ & $6(1)$ & $-4(1)$ \\
\hline
\end{tabular}


Table 4. Bond lengths $[\AA]$ and angles $\left[^{\circ}\right]$.

\begin{tabular}{|c|c|c|c|}
\hline $\operatorname{Br}(1)-C(17)$ & $1.953(2)$ & $C(3)-C(8)$ & $1.391(3)$ \\
\hline $\mathrm{S}(1)-\mathrm{O}(2)$ & $1.434(2)$ & $C(4)-C(5)$ & $1.394(3)$ \\
\hline $\mathrm{S}(1)-\mathrm{O}(1)$ & $1.436(2)$ & $C(5)-C(6)$ & $1.390(3)$ \\
\hline $\mathrm{S}(1)-\mathrm{N}(1)$ & $1.639(2)$ & $C(6)-C(7)$ & $1.393(3)$ \\
\hline $\mathrm{S}(1)-\mathrm{C}(9)$ & $1.768(2)$ & $C(7)-C(8)$ & $1.393(3)$ \\
\hline $\mathrm{N}(1)-\mathrm{C}(8)$ & $1.427(2)$ & $\mathrm{C}(9)-\mathrm{C}(10)$ & $1.394(3)$ \\
\hline$N(1)-C(1)$ & $1.515(2)$ & $\mathrm{C}(9)-\mathrm{C}(14)$ & $1.397(3)$ \\
\hline $\mathrm{C}(1)-\mathrm{C}(17)$ & $1.519(3)$ & $\mathrm{C}(10)-\mathrm{C}(11)$ & $1.388(3)$ \\
\hline $\mathrm{C}(1)-\mathrm{C}(16)$ & $1.534(3)$ & $\mathrm{C}(11)-\mathrm{C}(12)$ & $1.396(3)$ \\
\hline$C(1)-C(2)$ & $1.553(3)$ & $\mathrm{C}(12)-\mathrm{C}(13)$ & $1.399(3)$ \\
\hline$C(2)-C(3)$ & $1.498(3)$ & $\mathrm{C}(12)-\mathrm{C}(15)$ & $1.507(3)$ \\
\hline$C(3)-C(4)$ & $1.384(3)$ & $\mathrm{C}(13)-\mathrm{C}(14)$ & $1.386(3)$ \\
\hline $\mathrm{O}(2)-\mathrm{S}(1)-\mathrm{O}(1)$ & & $C(4)-C(3)-C(8)$ & $120.3(2)$ \\
\hline $119.57(9)$ & & $C(4)-C(3)-C(2)$ & $129.0(2)$ \\
\hline $\mathrm{O}(2)-\mathrm{S}(1)-\mathrm{N}(1)$ & & $C(8)-C(3)-C(2)$ & $110.7(2)$ \\
\hline $106.39(9)$ & & $C(3)-C(4)-C(5)$ & $119.4(2)$ \\
\hline $\mathrm{O}(1)-\mathrm{S}(1)-\mathrm{N}(1)$ & & $C(6)-C(5)-C(4)$ & $119.9(2)$ \\
\hline $106.69(9)$ & & $C(5)-C(6)-C(7)$ & $121.3(2)$ \\
\hline $\mathrm{O}(2)-\mathrm{S}(1)-\mathrm{C}(9)$ & & $C(6)-C(7)-C(8)$ & $117.9(2)$ \\
\hline $107.60(9)$ & & $C(3)-C(8)-C(7)$ & $121.2(2)$ \\
\hline $\mathrm{O}(1)-\mathrm{S}(1)-\mathrm{C}(9)$ & & $\mathrm{C}(3)-\mathrm{C}(8)-\mathrm{N}(1)$ & $109.8(2)$ \\
\hline $107.01(9)$ & & $\mathrm{C}(7)-\mathrm{C}(8)-\mathrm{N}(1)$ & $129.0(2)$ \\
\hline $\mathrm{N}(1)-\mathrm{S}(1)-\mathrm{C}(9)$ & & $C(10)-C(9)-C(14)$ & $120.1(2)$ \\
\hline $109.33(9)$ & & $\mathrm{C}(10)-\mathrm{C}(9)-\mathrm{S}(1)$ & $120.2(2)$ \\
\hline $\mathrm{C}(8)-\mathrm{N}(1)-\mathrm{C}(1)$ & $110.3(2)$ & $C(14)-C(9)-S(1)$ & $119.6(2)$ \\
\hline $\mathrm{C}(8)-\mathrm{N}(1)-\mathrm{S}(1)$ & $123.1(1)$ & $C(11)-C(10)-C(9)$ & $119.5(2)$ \\
\hline $\mathrm{C}(1)-\mathrm{N}(1)-\mathrm{S}(1)$ & $123.9(1)$ & $\mathrm{C}(10)-\mathrm{C}(11)-\mathrm{C}(12)$ & $121.4(2)$ \\
\hline $\mathrm{N}(1)-\mathrm{C}(1)-\mathrm{C}(17)$ & $111.1(2)$ & $\mathrm{C}(11)-\mathrm{C}(12)-\mathrm{C}(13)$ & $118.1(2)$ \\
\hline $\mathrm{N}(1)-\mathrm{C}(1)-\mathrm{C}(16)$ & $112.8(2)$ & $\mathrm{C}(11)-\mathrm{C}(12)-\mathrm{C}(15)$ & $121.8(2)$ \\
\hline$C(17)-C(1)-C(16)$ & $107.1(2)$ & $C(13)-C(12)-C(15)$ & $120.1(2)$ \\
\hline $\mathrm{N}(1)-\mathrm{C}(1)-\mathrm{C}(2)$ & $103.2(2)$ & $\mathrm{C}(14)-\mathrm{C}(13)-\mathrm{C}(12)$ & $121.3(2)$ \\
\hline $\mathrm{C}(17)-\mathrm{C}(1)-\mathrm{C}(2)$ & $112.9(2)$ & $\mathrm{C}(13)-\mathrm{C}(14)-\mathrm{C}(9)$ & $119.5(2)$ \\
\hline$C(16)-C(1)-C(2)$ & $109.9(2)$ & $\mathrm{C}(1)-\mathrm{C}(17)-\mathrm{Br}(1)$ & $114.0(2)$ \\
\hline$C(3)-C(2)-C(1)$ & $105.5(2)$ & & \\
\hline
\end{tabular}




\section{References.}

1. SMART and SAINTPLUS, Area detector control and integration software, Ver. 6.01. Bruker Analytical X-ray Systems, Madison, Wisconsin, U.S.A, 1999.

2. SHELXTL, An integrated system for solving, refining and displaying crystal structures from diffraction data, Ver. 5.10. Bruker Analytical X-ray Systems, Madison, Wisconsin, U.S.A, 1997.

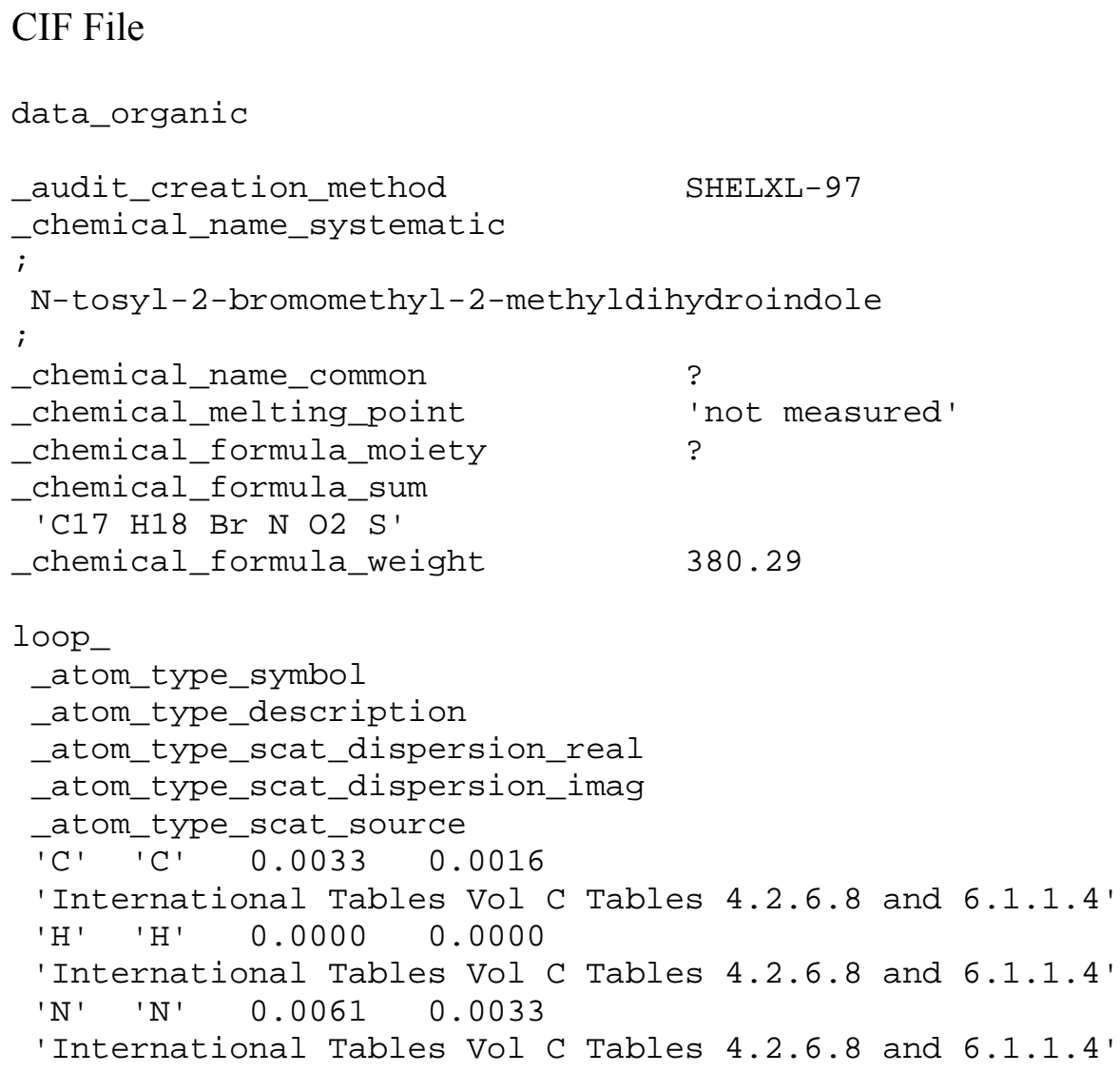




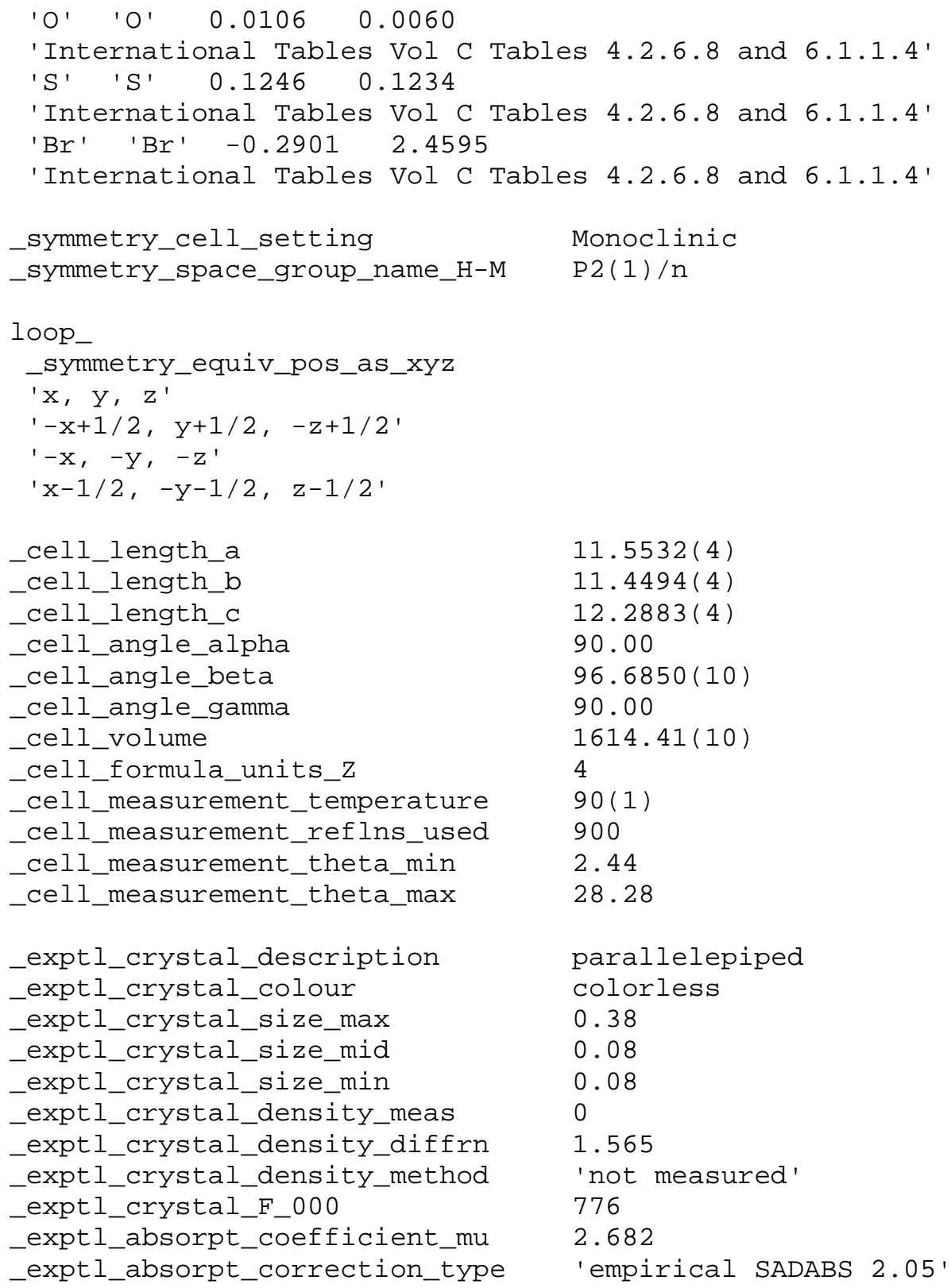

0.08

0.08

0

1.565

' not measured'

776

2.682

'empirical SADABS 2.05' 


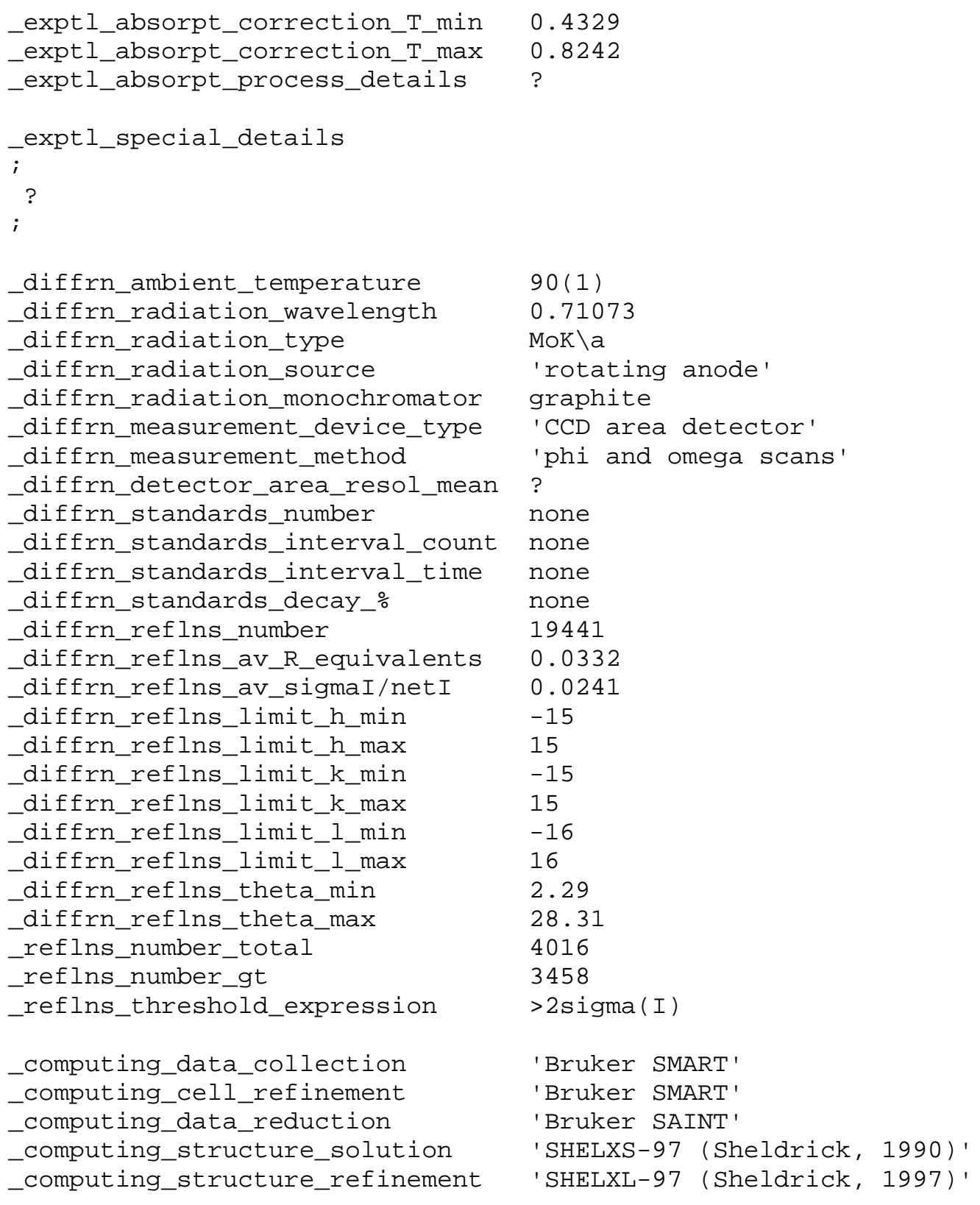




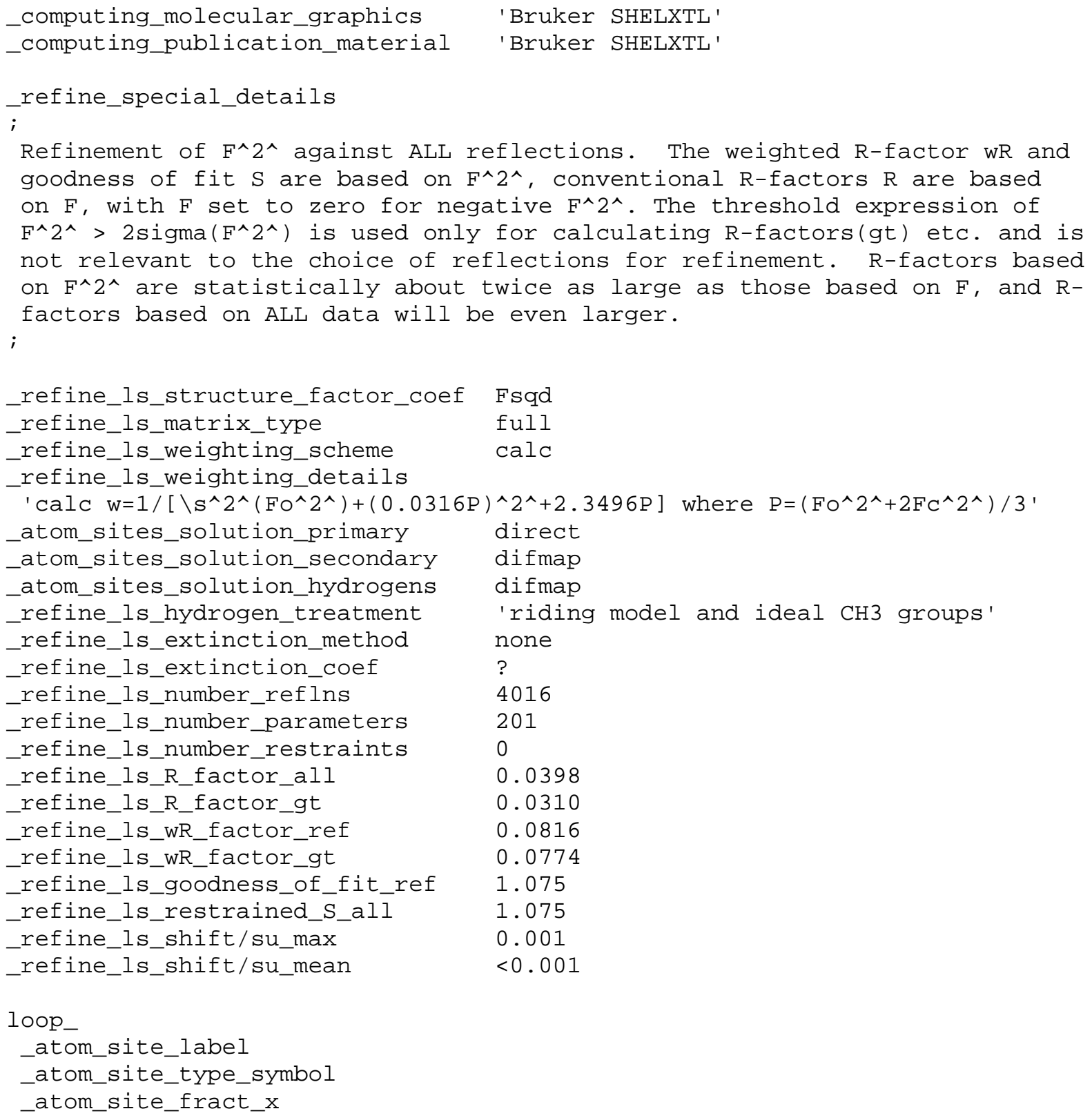




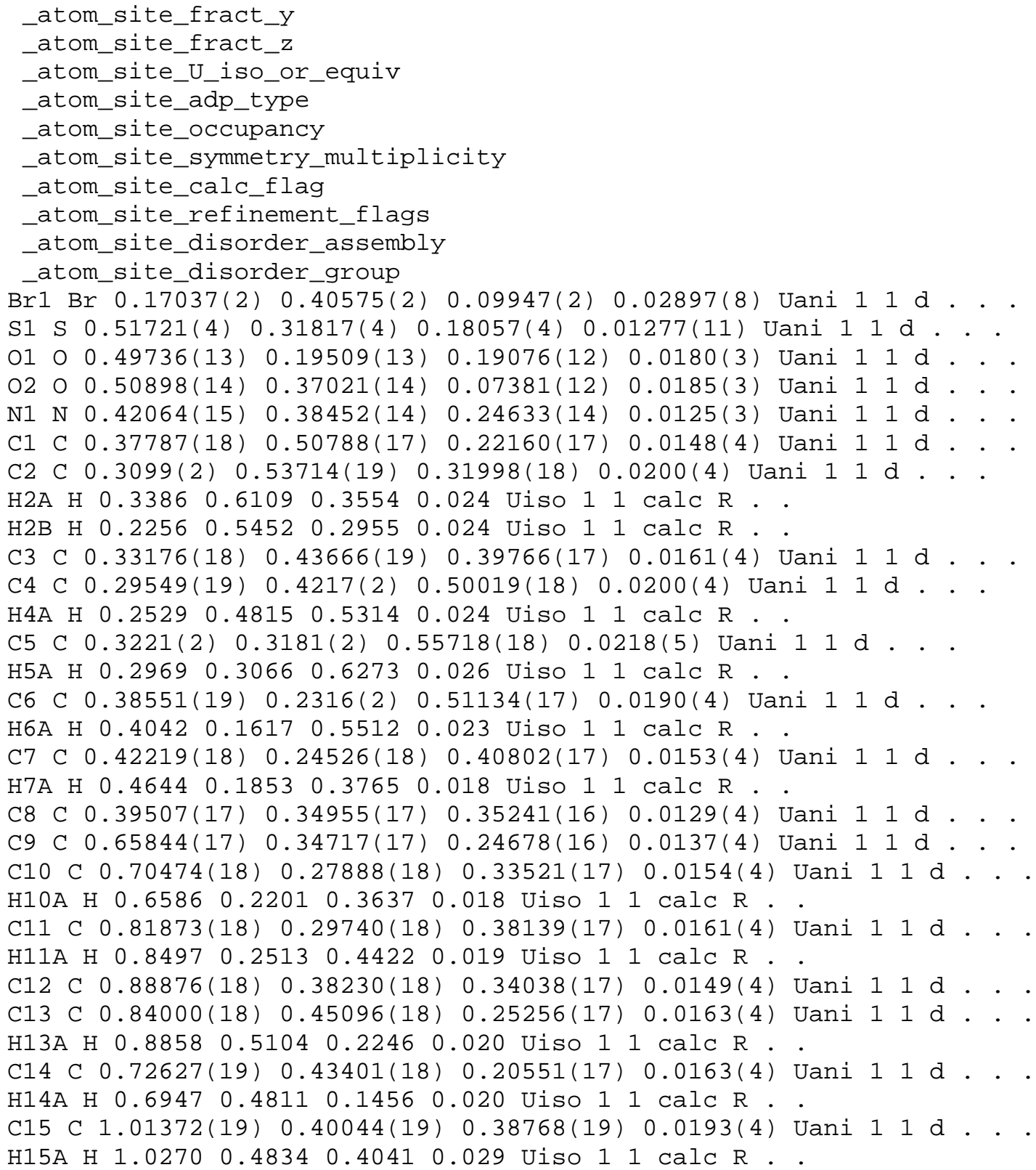


H15B H 1.06550 .37490 .33450 .029 Uiso 11 calc $\mathrm{R}$.

H15C H $1.0300 \quad 0.35480 .45520 .029$ Uiso 1 calc R .

C16 C $0.4777(2) \quad 0.5954(2) \quad 0.2166(2) \quad 0.0257$ (5) Uani 11 d . .

H16A H $0.51780 .5787 \quad 0.15230 .039$ Uiso 1 l calc R .

H16B H 0.53290 .58850 .28310 .039 Uiso 11 calc $R$.

H16C H $0.4460 \quad 0.67490 .21120 .039$ Uiso 11 calc R .

C17 C $0.30210(19) \quad 0.5137(2) \quad 0.11211(18) \quad 0.0208$ (4) Uani 11 d . .

H17A H $0.35100 .49660 .0530 \quad 0.025$ Uiso 11 calc R .

H17B H 0.27200 .59430 .10080 .025 Uiso 11 calc R .

loop_

_atom_site_aniso_label
-atom_site_aniso_U_11
_atom_site_aniso_U_22
-atom_site_aniso_U_33
-atom_site_aniso_U_23
-atom_site_aniso_U_13
_atom_site_aniso_U_12

$\overline{B r} 10 . \overline{0} 1817(12) \quad 0.0 \overline{2} 960(14) \quad 0.03676(15)-0.00948(10)-0.00685(9) \quad-0.00181(9)$

S1 $0.0154(2) \quad 0.0118(2) \quad 0.0109(2)-0.00149(17) \quad 0.00093(17) \quad 0.00002(17)$

$010.0211(8) \quad 0.0118(7) \quad 0.0207(7)-0.0040(6) \quad 0.0016(6)-0.0005(6)$

$020.0212(7) \quad 0.0231(8) \quad 0.0109(7) \quad 0.0003(6) \quad 0.0003(6) \quad-0.0001(6)$

$\begin{array}{lllllllll}\mathrm{N} 1 & 0.0154(8) & 0.0095(7) & 0.0126(8) & 0.0018(6) & 0.0018(6) & 0.0023(6)\end{array}$

C1 $0.0158(9) \quad 0.0102(9) \quad 0.0178(10) \quad 0.0020(7) \quad-0.0013(7) \quad 0.0011(7)$

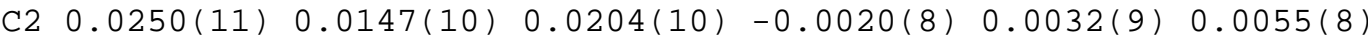

C3 $0.0152(9) \quad 0.0161(9) \quad 0.0166(10)-0.0023(8) \quad 0.0001(8)-0.0016(8)$

$\begin{array}{lllllll}C 4 & 0.0169(10) & 0.0250(11) & 0.0185(10) & -0.0075(9) & 0.0041(8) & -0.0021(8)\end{array}$

C5 $0.0196(10) \quad 0.0326(12) \quad 0.0131(9) \quad 0.0004(9) \quad 0.0020(8)-0.0101(9)$

$\begin{array}{lllllll}\mathrm{C} 6 & 0.0169(10) & 0.0230(11) & 0.0166(10) & 0.0070(8) & -0.0009(8) & -0.0072(8)\end{array}$

$\begin{array}{lllllll}C 7 & 0.0136(9) & 0.0158(9) & 0.0165(9) & 0.0029(8) & 0.0013(7) & -0.0014(7)\end{array}$

C8 $0.0122(9) \quad 0.0140(9) \quad 0.0120(9) \quad 0.0001(7)-0.0004(7)-0.0024(7)$

C9 $0.0150(9) \quad 0.0136(9) \quad 0.0127(9) \quad-0.0018(7) \quad 0.0023(7) \quad 0.0021(7)$

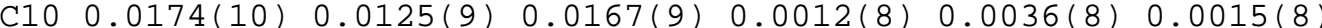

$\begin{array}{lllllll}\mathrm{C} 11 & 0.0168(9) & 0.0147(9) & 0.0167(9) & 0.0013(7) & 0.0018(8) & 0.0027(8)\end{array}$

$\begin{array}{lllllll}\mathrm{C} 12 & 0.0157(9) & 0.0130(9) & 0.0165(9) & -0.0042(7) & 0.0032(8) & 0.0023(7)\end{array}$

$\begin{array}{lllllll}\mathrm{C} 13 & 0.0186(10) & 0.0130(9) & 0.0183(10) & -0.0004(8) & 0.0056(8) & -0.0012(8)\end{array}$

$\begin{array}{lllllll}\mathrm{C} 14 & 0.0198(10) & 0.0149(9) & 0.0146(9) & 0.0021(7) & 0.0030(8) & 0.0010(8)\end{array}$

$\begin{array}{lllllll}\mathrm{C} 15 & 0.0161(10) & 0.0170(10) & 0.0245(11) & -0.0009(8) & 0.0011(8) & -0.0006(8)\end{array}$

$\begin{array}{lllllll}\mathrm{C} 16 & 0.0174(10) & 0.0169(11) & 0.0425(14) & 0.0091(10) & 0.0019(10) & 0.0007(8)\end{array}$

$\begin{array}{lllllll}\mathrm{C} 17 & 0.0161(10) & 0.0247(11) & 0.0203(10) & 0.0058(9) & -0.0038(8) & 0.0005(8)\end{array}$ 
All esds (except the esd in the dihedral angle between two l.s. planes) are estimated using the full covariance matrix. The cell esds are taken into account individually in the estimation of esds in distances, angles and torsion angles; correlations between esds in cell parameters are only used when they are defined by crystal symmetry. An approximate (isotropic) treatment of cell esds is used for estimating esds involving l.s. planes. ;

loop_

_geom_bond_atom_site_label_1 -geom_bond_atom_site_label_2 -geom_bond_distance

_geom_bond_site_symmetry_2

_geom_bond_publ_flag

Br1 C17 1.953(2) . ?

S1 O2 1.4339(15) . ?

S1 $011.4355(15)$. ?

S1 N1 1.6385(17) . ?

S1 C9 1.768(2) . ?

N1 C8 $1.427(2)$. ?

N1 C1 $1.515(2)$. ?

C1 C17 1.519(3) . ?

C1 C16 1.534(3) . ?

C1 C2 $1.553(3)$. ?

C2 C3 $1.498(3)$. ?

C3 C4 1.384(3) . ?

C3 C8 $1.391(3)$. ?

C4 C5 1.394(3) . ?

C5 C6 $1.390(3)$. ?

C6 C7 1.393(3) . ?

C7 C8 1.393(3) . ?

C9 C10 1.394(3) . ?

C9 C14 1.397(3) . ?

C10 C11 $1.388(3)$. ?

C11 C12 $1.396(3)$. ?

$\mathrm{C} 12 \mathrm{C} 131.399(3)$. ?

C12 C15 1.507(3) . ?

C13 C14 1.386(3) . ? 
loop_

_geom_angle_atom_site_label_1 geom angle atom site label_2 _geom_angle_atom_site_label_3 -geom_angle

_geom_angle_site_symmetry_1 -geom_angle_site_symmetry_3 _geom_angle_publ_flag

O2 S1 01 119.57(9) . . ?

O2 S1 N1 106.39(9) . . ?

O1 S1 N1 106.69(9)..?

02 S1 C9 $107.60(9)$. . ?

O1 S1 C9 $107.01(9) . . ?$

N1 S1 C9 $109.33(9)$. . ?

C8 N1 C1 110.33(16) . . ?

C8 N1 S1 123.07(14) . . ?

C1 N1 S1 123.92(13) . . ?

N1 C1 C17 111.09(17) . . ?

N1 C1 C16 112.81(17) . . ?

C17 C1 C16 107.10(18) . . ?

N1 C1 C2 103.16(16) . . ?

C17 C1 C2 112.86(18) . . ?

C16 C1 C2 109.92(18) . . ?

C3 C2 C1 105.51(17) . . ?

C4 C3 C8 120.3(2) . . ?

$\begin{array}{llllllll}C 4 & \text { C3 } & \text { C2 } & 129.0(2) & \text {. . ? }\end{array}$

C8 C3 C2 $110.71(18)$. . ?

C3 C4 C5 $119.4(2)$. ? ?

C6 $\mathrm{C} 5 \mathrm{C} 4 \mathrm{119.9(2)..?}$

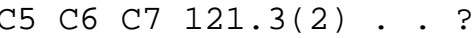

C6 C7 C8 $117.9(2)$. . ?

C3 C8 C7 121.15(19) . . ?

C3 C8 N1 109.79(17) . . ?

C7 C8 N1 129.04(18) . . ?

C10 C9 C14 120.10(19) . . ?

C10 C9 S1 $120.22(16)$. . ?

C14 C9 S1 119.55(16) . . ?

C11 C10 C9 $119.52(19) . . ?$

C10 C11 C12 121.39(19) . . ?

C11 C12 C13 118.13(19) . . ?

C11 C12 C15 121.76(19) . . ? 


\begin{abstract}
C13 C12 C15 120.11(19) . . ?
C14 C13 C12 121.32(19) . . ?

C13 C14 C9 $119.52(19) . . ?$

C1 C17 Br1 113.95(15) . . ?
\end{abstract}

loop_

_geom_torsion_atom_site_label_1 geom torsion atom site_label_2 _geom_torsion_atom_site_label_3 -geom_torsion_atom_site_label_4 -geom_torsion

_geom_torsion_site_symmetry_1 -geom_torsion_site_symmetry_2 _geom_torsion_site_symmetry_3 -geom_torsion_site_symmetry_4 -geom_torsion_publ_flag O 2 S1 N1 C8 $174.05(15) . .$. ? O1 S1 N1 C8 $45.36(18)$. . . . ?

C9 S1 N1 C8 $-70.03(18) . .$. ?

O2 S1 N1 C1 $-26.32(18)$. . . ?

O1 S1 N1 C1 $-155.01(15)$. . . ?

C9 S1 N1 C1 $89.60(17) . . . \quad$. ?

C8 N1 C1 C17

S1 N1 C1 C17 $70.2(2)$. . . . ?

C8 N1 C1 C16 111.8(2) • . . . ?

S1 N1 C1 C16 $-50.0(2) \cdot . \quad . \quad . \quad ?$

C8 N1 C1 C2 $-6.7(2)$.

S1 N1 C1 C2 $-168.58(14)$. . . ?

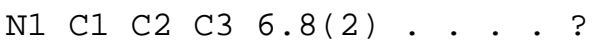

C17 C1 C2 C3 126.79(19) . . . . ?

C16 C1 C2 C3 $-113.72(19) . . . . ?$

C1 C2 C3 C4 $176.8(2) . . \quad . \quad . \quad$ ?

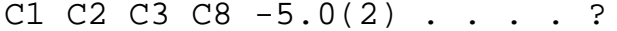

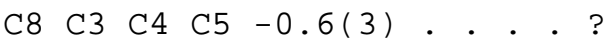

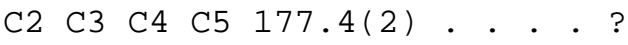

C3 $\mathrm{C} 4 \mathrm{C} 5 \mathrm{C} 6 \quad 0.7(3) \cdot . \quad \cdot \quad . \quad$ ?

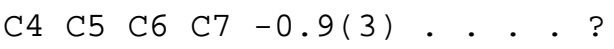

C5 $\mathrm{C} 6 \mathrm{C} 7 \mathrm{C} 8 \mathrm{1.1}(3) \cdot . \cdot . \quad$ ?

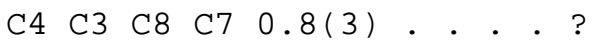

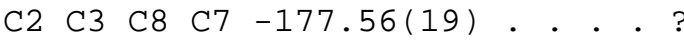

C4 C3 C8 N1 179.17(18) . . . ? 


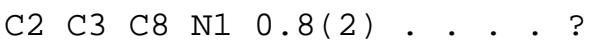

C6 C7 C8 C3 $-1.0(3)$. . . . ?

C6 C7 C8 N1 -179.05(19) . . . ?

C1 N1 C8 C3 3.9(2) . . . . ?

S1 N1 C8 C3 $166.00(15)$. . . . ?

C1 N1 C8 C7 $-177.85(19) \quad . \quad . \quad . \quad$ ?

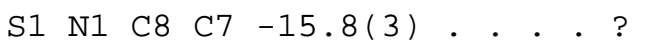

O2 S1 C9 C10 $-159.74(16) \cdot \cdot \cdot \cdot$ ?

O1 S1 C9 C10 $-30.07(19)$. . . . ?

N1 S1 C9 C10 85.12(18) . . . . ?

O2 S1 C9 C14 16.12(19) . . . . ?

O1 S1 C9 C14 $145.79(16)$. . . ?

N1 S1 C9 C14 $-99.02(17) \quad . \quad . \quad . \quad$ ?

C14 C9 C10 C11 $-0.4(3)$. . . ?

S1 C9 C10 C11 175.48(16) . . . ?

C9 $\mathrm{C} 10 \mathrm{C} 11 \mathrm{C} 12-0.7(3) \quad . \quad . \quad . \quad . \quad$ ?

C10 C11 C12 C13 $1.6(3) . . . . ?$

C10 C11 C12 C15 -178.03(19) . . . ?

C11 C12 C13 C14

C15 C12 C13 C14 178.21(19) . . . ?

$\mathrm{C} 12 \mathrm{C} 13 \mathrm{C} 14 \mathrm{C} 90.4(3)$. . . . ?

C10 C9 C14 C13 $0.5(3)$. . . . ?

S1 C9 C14 C13 $-175.34(16) \quad . \quad . \quad . \quad$ ?

N1 C1 C17 Br1 55.1(2) . . . . ?

C16 C1 C17 Br1 178.75(15) . . . ?

C2 $\mathrm{C} 1 \mathrm{C} 17 \mathrm{Br} 1-60.1(2) . . . \quad$ ?

_diffrn_measured_fraction_theta_max _diffrn_reflns_theta_full

_diffrn_measured_fraction_theta_full

0.997

28.31

_refine_diff_density_max 1.499

_refine_diff_density_min $\quad-0.615$

_refine_diff_density_rms 0.092 